\title{
Analysis of Hepatobiliary Disorder Reports Associated With the Use of Herbal Medicines in the Global Suspected ADR Database Vigibase
}

\begin{abstract}
Florence van Hunsel ${ }^{1 *}$, Sonja van de Koppel ${ }^{1}$, Souad Skalli ${ }^{2}$, Andrea Kuemmerle ${ }^{3,4}$, Lida Teng ${ }^{5}$, Jia-bo Wang ${ }^{6}$ and Joanne Barnes ${ }^{7}$, on behalf of the International Society of Pharmacovigilance Herbal Traditional Medicines Special Interest Group

${ }^{1}$ The Netherlands Pharmacovigilance Centre Lareb, 's-Hertogenbosch, Netherlands, ${ }^{2}$ Microbiology and Molecular Biology Team, Center of Plant and Microbial Biotechnology, Biodiversity and Environment, Faculty of Sciences, Mohammed V University in Rabat, Rabat, Morocco, ${ }^{3}$ Department of Medicine, Swiss Tropical and Public Health Institute, Basel, Switzerland, ${ }^{4}$ University of Basel, Basel, Switzerland, ${ }^{5}$ Department of Health Economics and Outcomes Research, Graduate School of Pharmaceutical Sciences, The University of Tokyo, Tokyo, Japan, ${ }^{6}$ Institute of Chinese Herbal Medicine, Beijing Integrative Medical Center for Liver Diseases, Beijing 302 Hospital of China, Beijing, China, ${ }^{7}$ School of Pharmacy, The University of Auckland, Auckland, New Zealand
\end{abstract}

\section{OPEN ACCESS}

Edited by: Anthony Booker,

University of Westminster, United Kingdom

Reviewed by: Hung-Rong Yen,

China Medical University, Taiwan Elena Y. Enioutina, The University of Utah, United States

${ }^{*}$ Correspondence: Florence van Hunsel f.vanhunse/@lareb.n

Specialty section: This article was submitted to Ethnopharmacology, a section of the journal

Frontiers in Pharmacology

Received: 27 June 2019 Accepted: 15 October 2019 Published: 06 November 2019

Citation:

van Hunsel F, van de Koppel S, Skalli S, Kuemmerle A, Teng L, Wang J-b and Barnes J (2019) Analysis of Hepatobiliary Disorder Reports Associated With the Use of Herbal Medicines in the Global Suspected ADR Database Vigibase.

Front. Pharmacol. 10:1326. doi: 10.3389/fphar.2019.01326
Introduction: Use of herbal medicines (HMs) is widespread across the world, with many people relying on $\mathrm{HMs}$ for their primary healthcare or using HMs in the context of a healthy life style. HMs originate from plant material and, as such, are often seen as "natural" and believed to be (relatively) safe by patients. Hepatobiliary disorders have been associated with numerous HMs.

Aim: This paper aims to analyze reporting patterns for hepatobiliary disorders associated with HMs use from reports submitted to the WHO global database of individual case safety reports (ICSRs) VigiBase.

Methods: A data extraction in VigiBase, the WHO international database of ICSR reports, was performed by the Uppsala Monitoring Centre on 2019-01-16. The dataset contained all ICSRs where an HM was identified with the UMC-assigned ATC code "V90: unspecified herbal and traditional medicine" and where the HM was classified as being either the suspected drug or an interacting drug, and containing at least one adverse reaction in the MedDRA ${ }^{\circledR}$ System Organ Class (SOC) Hepatobiliary Disorders (HBD). Descriptive analyses in Excel $2013^{\circledR}$ were used to determine general characteristics of the reports in the broad data set, including total number of reports, reporting country and patient characteristics. For single suspect herbal reports, reports categorized as "serious" according to CIOMS criteria (CIOMS), 2001) were extracted.

Results: In total, 2,483 reports describing with at least one ADR in the SOC HBD were extracted from VigiBase. In total, 780 (31.4\%) reports concern only one suspect HM. However, for 188 reports of these reports (24.1\%), the single suspect herbal preparation contains more than one herbal ingredient. The 592 reports for single suspect herbal preparations described a total of 764 ADRs in the SOC HBD. Jaundice was the most reported ADR for these reports. 
Conclusion: Almost 2,500 reports for HMs and with at least one ADR coded to the MedDRA ${ }^{\circledR}$ SOC HBD were retrieved from VigiBase. Of the HBD SOC HM reports, around $25 \%$ concerned a single herbal species as the suspect "drug." Substantial issues with coding of the suspect herbal drugs were found. In-depth causality assessment of the cases is needed to draw conclusions on the strength of the relationships.

Keywords: herbal medicines, hepatobiliary disorders, adverse drug reactions, VigiBase, pharmacovigilance

\section{INTRODUCTION}

Herbal medicines (HMs) include herbs, herbal materials, herbal preparations (comminuted or powdered herbal materials, or extracts, tinctures and fatty oils of herbal materials) and finished or manufactured herbal products found in pharmaceutical dosage forms (tablets, capsules) (World Health Organization, 2004). Traditional medicine collectively refers to numerous different medical systems [e.g., Traditional Chinese medicine (TCM), Ayurvedic medicine, Australian Aboriginal medicine, medical herbalism, etc] that has a long history of use. It is the sum total of the knowledge, skills, and practices based on the theories, beliefs, and experiences indigenous to different cultures, whether explicable or not, used in the maintenance of health, as well as in the prevention, diagnosis, improvement or treatment of physical and mental illnesses. Most traditional medical systems make use of medicinal plants and, sometimes, other natural substances (e.g., animal parts, minerals) as a/the main approach to medical treatment. The terms complementary/alternative/nonconventional medicine are used interchangeably with traditional medicine in some countries (World Health Organization, 2004). Use of HMs and other TMs is widespread across the world, with many people relying on HMs/TMs for their primary healthcare, particularly those in low-middle income countries (WHO TCAM strategy) (World Health Organization, 2013), or using HMs in the context of a healthy life style (Ekor, 2014). The use of HMs in Europe is also widespread (Fisher and Ward, 1994; Barnes et al., 1999; de Boer et al., 2015; Kemppainen et al., 2018). HMs originate from plant material and, as such, are often seen as "natural" and believed to be (relatively) safe by patients (Vickers et al., 2006). However, HMs contain pharmacologically active constituents, and numerous HMs, or their constituents, have been associated with the occurrence of adverse drug reactions (ADRs) (Posadzki et al., 2013; de Boer et al., 2015; Venhuis et al., 2016). The occurrence of ADRs associated with HMs, including those resulting from interactions between herbal medicines (HMs) and conventional drugs, is a global public health concern (Skalli et al., 2007; Skalli and Soulaymani, 2012). Against this background, pharmacovigilance (PV) activities to identify, evaluate, and respond to signals of safety concerns associated with HMs are essential (Barnes, 2003).

PV is defined as "the science and activities relating to the detection, assessment, understanding and prevention of adverse effects or any other drug-related problem" (World Health Organization, 2019). The World Health Organization (WHO) established its Programme for International Drug Monitoring in response to the thalidomide disaster detected in 1961. Together with the WHO Collaborating
Centre for International Drug Monitoring (the Uppsala Monitoring Centre in Sweden), the WHO promotes PV activities at the country level (the World Health Organization, 2019). The UMC maintains the global ADR database of individual case safety reports (ICSRs) VigiBase. This database contains over 19 million reports of ADRs related to medicines, submitted, since 1968 , by member countries of the WHO Programme for International Drug Monitoring (the Uppsala Monitoring Centre, 2019).

Spontaneous ADR reporting and active surveillance conducted by national PV centers can permit rapid detection of potentially harmful combinations of medicines and HMs (Skalli and Soulaymani, 2012). However, while PV systems for conventional medicines are well-established and incorporated in healthcare and regulatory activities, this is far less the case for HMs (Barnes, 2003).

In undertaking PV for HMs, the complex characteristics of HMs, not least that each herbal ingredient typically contains hundreds of chemical constituents, batch-to-batch chemical variation of herbal ingredients and common place use of ambiguous nomenclature pose particular challenges (Dauncey et al., 2019; Shaw et al., 2012). Further difficulties arise due to the ways in which many herbal products are formulated (for example, as multi-ingredient products containing other herbal or non-herbal substances), used by patients (for example, concurrent use of multiple HM products or together with conventional drugs), and used by herbal or traditional medicine practitioners (for example, as individualized, multi-ingredient formulae, which may change with each consultation) (Barnes, 2003). A recognized limitation of spontaneous reports is that the incidence and prevalence of ADRs related to medicines are difficult to predict because of under-reporting (Hazell and Shakir, 2006); for several reasons, this is likely to be even greater for HMs than for conventional medicines (Barnes et al., 1998; Skalli and Soulaymani, 2012; Vohra et al., 2012; Necyk et al., 2014). Despite these issues, spontaneous reporting of ADRs remains the cornerstone of PV for HMs and can be used to gain insight into the safety of HMs as used in clinical practice (Skalli and Soulaymani, 2012).

Among the risks associated with HMs, hepatobiliary disorders (HBD) have been associated with numerous HMs (Real et al., 2019). HBDs are non-neoplastic or neoplastic disorders that affect the liver, bile ducts, and/or gallbladder. Representative examples of non-neoplastic disorders include hepatitis, cirrhosis, and cholangitis (The SIDER Side Effect Resource, 2015). Recognition of HBD is increasing, and the list of HMs implicated in causing these reactions has continued to grow and is of international interest (Navarro et al., 2017; Real et al., 2019). In some countries in the Asia-Pacific region, HMs and "dietary supplements" are major causes of HBD (Wong et al., 2019). Dietary supplements are often defined as products taken 
by mouth that contain a "dietary ingredient." Dietary ingredients include vitamins, minerals, amino acids, and herbs or botanicals, as well as other substances that can be used to supplement the diet. Dietary supplements come in many dose forms, including tablets, capsules, powders, energy bars, and liquids (US Food and Drug Administration, 2015). A large systematic review, aiming to evaluate the clinical characteristics and outcomes of TCM-induced liver injury and to estimate the proportion of TCM-related liver injury in all drug-induced liver injuries, showed that in China, around 25\% of TCMs are suspected drugs in published HBD cases. This proportion has gradually increased between 1998 and 2016 (Wang et al., 2018b). HM-related hepatotoxicity is also a common cause of drug-induced liver injury in Western countries (Stournaras and Tziomalos, 2015). In a prospective study from Iceland that included 96 patients with drug-induced liver injury between 2010 and 2011, 16\% of cases were attributable to dietary supplements (Björnsson et al., 2013). The drug induced liver injury network in the United States, which upholds the largest database of HMs-related hepatotoxicity, found that between 2004 and 2013, 15.5\% of cases (130 out of 839 in total) of DILI were likely caused by herbal dietary supplements. In addition, they found a temporal trend in increase in liver injury herbal dietary supplements (Navarro et al, 2014).

HMs associated with HBD include green tea (Camellia sinensis) extracts, ephedra (Ephedra sinica, also known as ma huang), black cohosh (Actaea racemosa), germander (Teucrium chamaedrys), kava (kava kava or Piper methysticum), blue skullcap (Scutellaria lateriflora), pennyroyal (Mentha pulegium), and HMs containing unsaturated pyrrolizidine alkaloids (Bunchorntavakul and Reddy, 2013; Garcia-Cortes et al., 2016; Real et al., 2019). Some HMs contain illicit ingredients, such as androgenic anabolic steroids in supplements promoted for body building, which may be the cause of drug-induced liver injury (DILI) rather than the labeled herbal ingredients (Garcia-Cortes et al., 2016).

\section{AIM}

This paper aims to analyze reporting patterns for HBD associated with HMs from reports submitted to the WHO global database of ICSRs VigiBase.

\section{METHODS}

\section{Data Source and Definition Dataset}

A data extraction in VigiBase, the WHO international database of suspected ADR reports (Linquist, 2008), was performed by the Uppsala Monitoring Centre (UMC) on 2019-01-16. The dataset date was 2019-01-01. The extracted dataset contained all ICSRs where a HM was identified with the UMC-assigned ATC code "V90: unspecified herbal and traditional medicine" and where the HM was classified by the reporter as being either suspected or interacting (reports with only concomitant HM drugs were not extracted). This ATC code is assigned to: products containing herbal ingredients only; to mixed products containing herbal ingredients and non-herbal substances (including ingredients of animal origin, e.g., deer velvet; substances such as vitamins/ minerals, and occasionally conventional drugs-where such substances are co-ingredients with herbal substances, e.g., lowdose paracetamol with herbal ingredients in traditional Chinese cough/cold remedies). Mixed products that contain both herbal ingredients and conventional medicines in one product were excluded from the analysis.

Herbals are coded at national center level in reports. The level of coding can include common names, brandnames of products or botanical names. The reported herbs are coded to a "PreferredBaseName," which is the drug active ingredient in VigiBase, which uses botanical names if possible. This entails that the reported medication name could be a common name, e.g., St John's wort, which are then coded as Hypericum perforatum for the drug active ingredient. As there maybe multiple common names for the same botanical name, there is in theory a level of "assumption" in this coding. For our analysis, the "PreferredBaseName" in VigiBase was used. ADRs were coded by national centers contributing to VigiBase. All ICSRs in VigiBase are automatically coded with MedDRA, the Medical Dictionary for Regulatory Activities (MedDRA) coding system, which refers to a group of MedDRA terms belonging to a System Organ Class [The International Conference on Harmonisation of Technical Requirements for Registration of Pharmaceuticals for Human Use (ICH), 2017]. For this study, a subset of all HM reports based on the MedDRA System Organ Class (SOC) HBD was extracted from VigiBase.

Some other MedDRA SOCs contain ADR "preferred terms" relevant to hepatic injury, such as the terms "hepatic enzymes increased" and "alanine aminotransferase increased" in the SOC Investigations; these were not included in this analysis.

Data were extracted on: date entered in UMC database; reporting country; seriousness; patient death; report type; patient age and gender; outcome of the ADR; onset date of the ADR(s); suspect "drug"; status as suspect or concomitant drug's; start and stop dates for "drug"; "drug" dose -usage, -administration, -route; indication for use; ADR on MedDRA SOC, -PT and -LLT level; causality as mentioned in the report; dechallenge (action taken and outcome); rechallenge (action taken and outcome).

Suspected duplicate ICSRs in VigiBase can automatically be identified with VigiMatch, an algorithm that uses a statistical model to score pairs of reports, taking into account the amount of matching and mismatching information (Tregunno et al., 2014). However, despite this, the final dataset can still contain some duplicates of reports.

It is important to note that the information in VigiBase comes from a variety of sources, and the likelihood that the suspected $\mathrm{ADR}$ is drug-related is not the same in all cases (Uppsala Monitoring Centre, 2019).

\section{Data Analysis}

Descriptive analyses in Excel $2013^{\circ}$ were used to determine general characteristics of the reports in the broad data set, including total number of reports, reporting country and patient characteristics. As spontaneous ADR for reports for herbals can contain multiple suspect drugs, including conventional medicines, as co-reported suspects, subsequent analyses were undertaken based only on reports with a single suspect herbal drug; these reports were 
extracted with R Software for Statistical Computing version 3.3.2 (2016-10-31) (The R Foundation, 2019).

From these single suspect herbal reports, reports categorized as "serious" (The Council for International Organizations of Medical Sciences (resulting in death, or is life-threatening; requires inpatient hospitalization or prolongation of existing hospitalization; results in persistent or significant disability or incapacity; results in a congenital anomaly (birth defect); or is otherwise "medically significant") (CIOMS), 2001) were extracted. The top 10 most commonly reported herbal drugs and most commonly reported ADRs were analyzed. The analysis used the MedDRA Preferred Term level(PT) (The International Conference on Harmonisation of Technical Requirements for Registration of Pharmaceuticals for Human Use (ICH), 2017) and the HM name specified as "Preferred Base Name." As one report can contain multiple ADRs, some reported ADRs may refer to the same patient.

\section{RESULTS}

A flowchart of the separate analysis steps for HMs that had at least one ADR in the MedDRA ${ }^{\circ}$ SOC HBD and the resulting sets of data which were analyzed are shown in Figure 1.

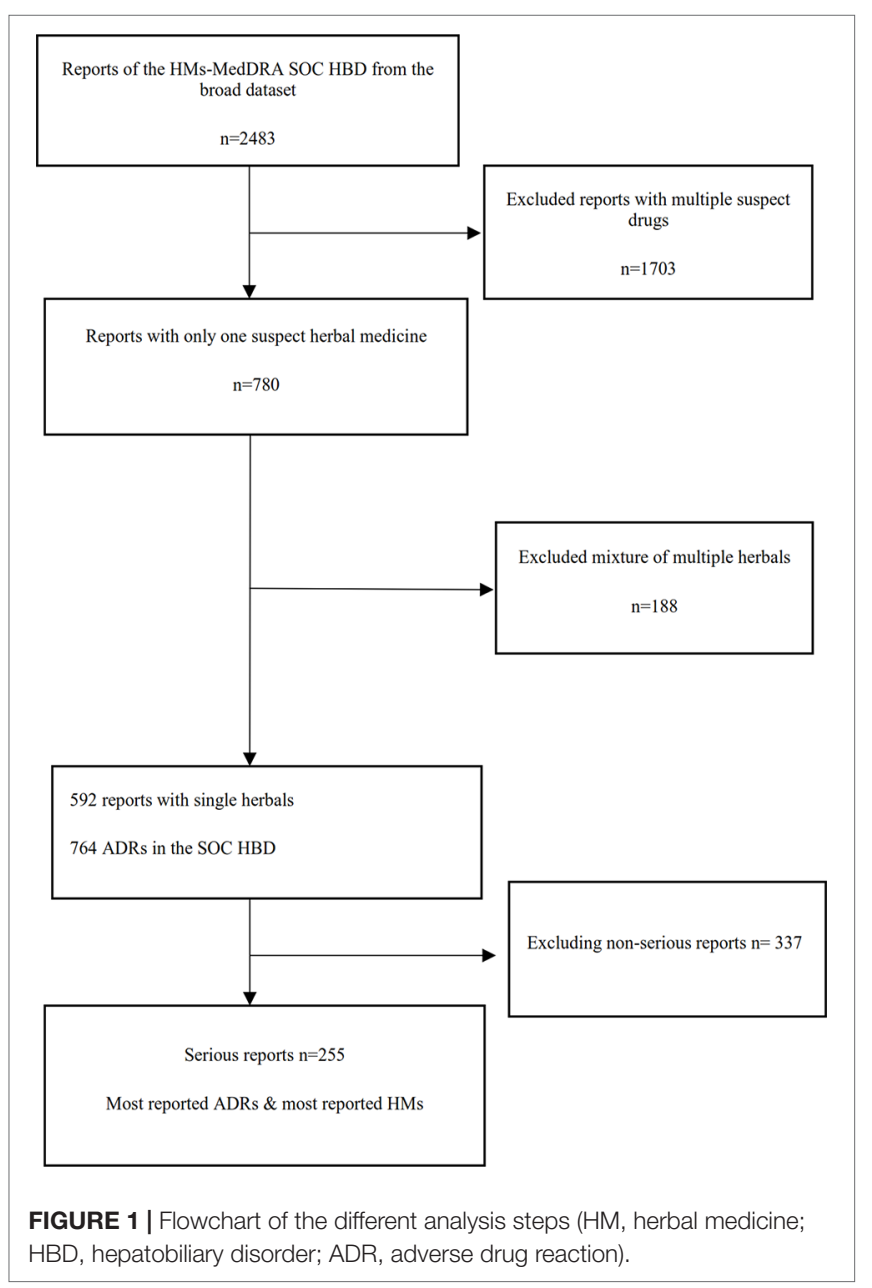

\section{Analysis of the Reports of the Broad Dataset General Overview}

In total, 2,483 reports for $\mathrm{HMs}$ with at least one $\mathrm{ADR}$ in the MedDRA SOC HBD were retrieved from VigiBase, dating from 31-03-1974 to 27-12-2018. This represents $0.013 \%$ of all reports in VigiBase. These reports were contributed by 49 countries. The number of reports per country varied; the most frequently reporting countries (top 10) contributed $82.6 \%(\mathrm{n}=2,051)$ of the total number of reports, and Japan submitted most reports ( $n=569 ; 22.9 \%$ of total), see Table 1. The number of reports submitted annually shows an increasing trend, with a peak in $2011(\mathrm{n}=293)$ reports.

\section{Description of the Reported Cases}

In total, 2,483 reported cases describing 46,447 ADRs were extracted from VigiBase for all SOCs. Of these, 13,738 ADRs concerned the SOC HBD. Most reports ( $\mathrm{n}=1032 ; 41.6 \%)$ were initially submitted by physicians; hospitals contributed $12.5 \%$, pharmacists $8.3 \%$, general practitioners $7.7 \%$, other health professionals $7.1 \%$, consumers or other non-health professionals $4.7 \%$, and specialist physicians $4 \%$. For 332 reports (13.3\%), the reporter was not known, or more than one profession was mentioned. In the reports, $63 \%$ of the patients was female, $35 \%$ male, and in $2 \%$, the gender was unknown. The mean reported age for women was 51 years (Median age 52 years) and mean age for men was 50 years (Median age 52 years). The reported age in one report was 220 years; this report was excluded from the analyses.

\section{Results of the Analysis for Single Suspect Herbal Medicines General Overview}

For most of the 2,483 reports, more than one herbal drug was reported as the suspect drug; 780 (31.4\%) reports concerned only one suspect HM. However, for 188 reports, the single suspect herbal preparation contains more than one herbal ingredient; these reports described a total of 335 ADRs. From the products containing multiple herbals Iberogast (which contains Angelica archangelica, Carum carvi, Chelidonium majus, Glycyrrhiza glabra, Iberis amara, Matricaria recutita, Melissa officinalis, Mentha piperita, Silybum marianum) was the most frequently reported ( $\mathrm{n}=10$ reports) herbal preparation.

For 592 reports with one suspect HM, the suspect herbal product concerned a single herbal species. Further analysis, reported below, was performed for these reports.

\section{Most Commonly Reported Single Suspect Herbals}

Table 2 lists the 10 most commonly reported HMs (by Preferred Base Name) reported in association with HBD SOC ADRs. The largest group, comprising 243 reports describing 309 ADRs, represents HMs or products in the database categorized as unspecified herbal [coded as Unspecified herbal, Ayurvedic preparation NOS, Herbal extract NOS, Herbal NOS, herbal preparation, unspecified herbal and traditional medicine, unspecified traditional medicine, herbal pollen NOS traditional medicine (Group NOS)]. 
TABLE 1 || Number of reports for herbal drugs submitted by the reporting countries

\begin{tabular}{|c|c|c|c|}
\hline Country & $\begin{array}{c}\text { Number of } \\
\text { reports }\end{array}$ & Country & $\begin{array}{c}\text { Number of } \\
\text { reports }\end{array}$ \\
\hline Japan & 569 & Turkey & 5 \\
\hline France & 295 & New Zealand & 5 \\
\hline Germany & 294 & Ireland & 5 \\
\hline USA & 211 & Mexico & 4 \\
\hline Spain & 134 & Viet Nam & 3 \\
\hline Australia & 130 & Uruguay & 3 \\
\hline UK & 117 & Brazil & 3 \\
\hline Switzerland & 114 & Greece & 3 \\
\hline India & 95 & Croatia & 2 \\
\hline Singapore & 92 & Iceland & 2 \\
\hline Morocco & 70 & Ghana & 2 \\
\hline Korea, Republic of & 63 & Kenya & 2 \\
\hline Canada & 53 & South Africa & 2 \\
\hline Malaysia & 40 & Nigeria & 2 \\
\hline Norway & 36 & Nepal & 1 \\
\hline Sweden & 23 & Finland & 1 \\
\hline China & 20 & Uganda & 1 \\
\hline Netherlands & 14 & Saudi Arabia & 1 \\
\hline Italy & 13 & Slovenia & 1 \\
\hline Thailand & 12 & Iraq & 1 \\
\hline Poland & 8 & Hungary & 1 \\
\hline Portugal & 7 & Russian Federation & 1 \\
\hline Belgium & 7 & Argentina & 1 \\
\hline Austria & 7 & Venezuela, Bolivarian Republic of & 1 \\
\hline Denmark & 6 & & \\
\hline Total 2,483 & & & \\
\hline
\end{tabular}

TABLE 2 | Ten most frequently reported single suspect HMs and number of ADRs per HM.

\begin{tabular}{|c|c|c|c|}
\hline & Herbal medicine & $\begin{array}{c}\text { Number of } \\
\text { reports }\end{array}$ & $\begin{array}{c}\text { Number of } \\
\text { ADRs }\end{array}$ \\
\hline 1 & Group non specified herbal drugs (nos) & 243 & 309 \\
\hline 2 & Chelidonium majus & 38 & 61 \\
\hline 3 & Cimicifuga racemosa & 31 & 49 \\
\hline 4 & Camellia sinensis & 21 & 25 \\
\hline 5 & Piper methysticum & 17 & 23 \\
\hline 6 & Valeriana officinalis & 16 & 19 \\
\hline 7 & Hypericum perforatum & 13 & 17 \\
\hline 8 & Teucrium chamaedrys & 12 & 13 \\
\hline $9=10$ & Senna siamea & 7 & 10 \\
\hline \multirow[t]{2}{*}{$9=10$} & Serenoa repens & 7 & 9 \\
\hline & Total & 405 & 535 \\
\hline
\end{tabular}

ADRs, adverse drug reactions; HM, herbal medicines.

\section{Most Commonly Reported ADRs}

The 592 reports for single suspect herbal preparations described a total of 1,368 ADRs. Since one drug, including herbal drugs, can be associated with multiple ADRs, and as these can relate to different organ systems, not all reported ADRs are within the HBD SOC. From the 1,368 reported ADRs, 764 MedDRA preferred terms were in the SOC HBD. Among these reports, jaundice was the most reported ADR (26\%) for reports concerning single suspect herbal drugs. The 10 most commonly reported HBD SOC ADRs are listed in Table 3.
TABLE 3 | Ten most commonly reported HBD SOC ADRs for reports with single suspect herbal medicines.

\begin{tabular}{llcc}
\hline & $\begin{array}{l}\text { Reported adverse drug } \\
\text { reaction (MedDRA PT name) }\end{array}$ & Number & $\begin{array}{c}\text { Percentage (\%) } \\
\text { top 10/total } \mathbf{~ = ~ 7 6 4 ~}\end{array}$ \\
\hline 1 & Jaundice & 146 & $26.4 / 19.1$ \\
2 & Hepatitis & 115 & $20.8 / 15.1$ \\
3 & Hepatic function abnormal & 90 & $16.3 / 11.8$ \\
4 & Drug-induced liver injury & 36 & $6.5 / 4.7$ \\
$5=5$ & Liver injury & 29 & $5.2 / 3.8$ \\
$5=6$ & Acute hepatic failure & 29 & $5.2 / 3.8$ \\
$5=7$ & Hepatic failure & 29 & $5.2 / 3.8$ \\
8 & Hepatitis cholestatic & 28 & $5.0 / 3.7$ \\
$9=9$ & Liver disorder & 26 & $4.7 / 3.4$ \\
$9=10$ & Hepatitis acute & 26 & $4.7 / 3.4$ \\
& Total & $\mathbf{5 5 4}$ & $\mathbf{1 0 0}$ \\
\hline
\end{tabular}

ADRs, adverse drug reactions; HBD, Hepatobiliary Disorder; SOC, System Organ Class.

\section{Results of the Analysis for "Serious" Reports Related to Single Suspect Herbal Medicines \\ General Overview}

In total, 255 (43\%) of the 592 reports for single suspect HMs were classified as serious. These reports concern 55 different HMs. For 127 reports, the herbal drug is specified (unspecified for 128 reports). In 119 reports (46.7\%) the indication was not filled in or PT "drug/product use for unknown indication" was reported. In the reports with a known indication; (unspecified) jaundice was reported 46 times (18\%). The top 10 reported indications concern obesity (reported 4 times (1.6\%)), menopause (reported 4 times $(1.6 \%)$ ), diabetes and abnormal weight gain (reported 3 times each (1.2\%)), migraine, rheumatoid arthritis, exfoliative dermatitis, slimming diet, climacteric discomfort (reported 2 times each $(0.8 \%))$. These 255 reports described 540 ADRs, with 329 ADRs coded to the MedDRA ${ }^{\circ}$ HBD SOC. In men, the most commonly reported ADRs were jaundice ( $\mathrm{n}=20$ reports), drug-induced liver injury $(\mathrm{n}=15)$ and hepatitis $(\mathrm{n}=13)$; among women, these were hepatic function abnormal and hepatitis ( $\mathrm{n}$ $=20$ for each $)$ and jaundice $(\mathrm{n}=18)$. Twenty-two reports were categorized as life threatening (with or without (prolonged) hospitalization) with the reported outcomes; 6 times recovered, one time recovered with sequel, 2 times recovering/resolving, 6 times not recovered, and 7 times not filled in or unknown. One hundred fifty reports were categorized as causing (prolonged) hospitalization where 44 times the patient recovered, 58 times was recovering, 28 times not recovered, 20 times the outcome is not been filled in or was unknown. Sixty-nine times the seriousness were categorized as "other" and 5 times the seriousness criterion was not filled in. In nine reports, the seriousness was categorized as "death." From those, one report has the outcome not recovered and eight reports described a fatal outcome. For "fatal" reports, the suspect HM was Rubia peregrina ( $\mathrm{n}=2)$; Hypericum perforatum, Valeriana officinalis, Senecio vulgaris, Andrographis paniculata, Piper methysticum and Cephaelis species were reported as the suspected drug for one report each. 


\section{Most Frequently Reported Herbals in Reports Classified as Serious}

The largest group of HMs among serious reports represented non-specified herbal products. Such products were described in 128 serious reports (50\%) for single suspect HM. Table 4 lists the 10 most commonly reported HMs (after exclusion of nonspecified herbal products) with at least more than one report per herbal.

Table 5 shows the ten most reported serious hepatic ADRs for the HMs (single suspect) listed in Table 4 (after exclusion of the "non-specified HMs" group).

\section{DISCUSSION}

This study explored the numbers and characteristics of hepatic ADR reports associated with HMs submitted to the global ICSR database VigiBase.

This analysis extracted 2,485 ICSRs for preparations coded as HMs that had at least one ADR in the MedDRA ${ }^{\circ}$ HBD SOC. These reports have been contributed by 49 countries since the 1970s; however, they may represent only a small proportion of the cases of liver injury associated with use of HMs. Under-reporting of ADRs associated with HMs is probably an even greater issue than for conventional medicines (Barnes, 2003; Walji et al., 2009; Skalli and Jordan, 2017; ). Often, healthcare professionals and users of HMs are unaware that spontaneous reporting systems accept reports associated with HMs and are unsure how to identify ADRs related to HMs. Also, many patients do not tell healthcare professionals that they use HMs, or may report "product complaints" to individuals, such as healthfood-store

TABLE 4 | Ten most commonly reported herbal drugs (after exclusion of nonspecified herbal products) among serious reports with ADRs coded to MedDRA HBD SOC.

\begin{tabular}{llcc}
\hline & Herbal medicine & Number of reports $(\mathbf{n}>\mathbf{1})$ & Number of ADRs \\
\hline 1 & Cimicifuga racemosa & 18 & 26 \\
2 & Valeriana officinalis & 14 & 17 \\
3 & Camellia sinensis & 9 & 11 \\
4 & Hypericum perforatum & 8 & 11 \\
4 & Serenoa repens & 5 & 7 \\
4 & Pelargonium sidoides & 4 & 4 \\
7 & Morinda citrifolia & 3 & 5 \\
7 & Aristolochia fontanesii & 3 & 3 \\
9 & Glycine max & 2 & 8 \\
9 & Chelidonium majus & 4 & 5 \\
9 & Arctostaphylos uva-ursi & 2 & 4 \\
9 & Hintonia latiflora & 2 & 4 \\
9 & Viscum album & 2 & 4 \\
9 & Aloe vera & 2 & 3 \\
9 & Petasites hybridus & 2 & 3 \\
9 & Ilex paraguariensis & 2 & 2 \\
9 & Rubia peregrina & 2 & 2 \\
9 & Lycium barbarum & 2 & 2 \\
9 & Silybum marianum & 2 & $\mathbf{1 2 3}$ \\
& Total & $\mathbf{8 8}$ & $5 v 5 t$
\end{tabular}

ADRs, adverse drug reactions; HBD, Hepatobiliary Disorder; SOC, System Organ Class. staff, outside the reporting framework (Walji et al., 2009). Thus, ADRs may go undetected and unreported to the formal system (Walji et al., 2009).

Under-reporting is a problem in all spontaneous reporting systems, and it can vary between countries: not all countries have been a part of the WHO Programme for International Drug Monitoring for the same amount of time, and some are selective about the type of reports they submit (Farah et al., 2000). Given the wide availability, use and acceptance of TCMs in China (Liu et al., 2015), the under-representation of ADR reports associated with HMs contributed from China in VigiBase demands comment. In China, ADRs are reported to the regional centers and then forwarded to the national center. By the end of year 2013 , the national system had collected $>6.6$ million reports. Numbers of ADR reports and ADR reporting rates for China have shown an increasing trend in recent years (Guo et al., 2015); Reports relevant to TCM increased from $13.3 \%$ in 2009 to $17.3 \%$ (Guo et al., 2015). However, the proportion of serious reports, as well as the proportions of reports contributed by consumers and pharmaceutical companies, remain low; importantly, in the context of HMs, a greater focus should be placed on reporting for TCMs, particularly TCM injections, some of which have been associated with serious ADRs (Guo et al., 2015). Also, these reports are not all represented in VigiBase. In 2018, approximately $5 \%$ of all reports in VigiBase were from China (Ploen, 2018) and our current analysis shows that China is not represented in the top 10 reporting countries for $\mathrm{HMs}$ with an ADR in the SOC HBD. From the literature, however, it is known that herbal TCM products can cause hepatotoxicity. An analysis of reported cases found numerous specified herbal TCM products with potential hepatotoxicity and, for several of these, causality seemed likely after further assessment (Teschke, 2014). Clinical outcomes of herbal TCM-induced hepatotoxicity can be serious, including liver transplantations or death (Wang et al., 2018b). The same issue (low numbers of reports for herbal hepatotoxicity in VigiBase) exists for other countries, for example, in Africa and India where the use of HM is also widespread (Skalli and Bencheikh, 2015; Gupta et al., 2017).

To investigate the association between HMs and reported $\mathrm{HBD}$ in a (spontaneous) reporting database such as VigiBase, comprehensive causality assessment is necessary (Meyboom et al., 1997). For HM-induced HBD cases, causality assessment should consider: confounding variables related to the documentation of the herbal product and the clinical course of the adverse event; hepatotoxicity and re-exposure criteria; temporal association; concomitant medication and alternative explanations, with special attention to pre-existing diseases of the liver, bile ducts and the pancreas (Teschke et al., 2013). The possibility of adulteration and/or contamination should also be considered (Wang et al., 2018a). Use of tools, such as RUCAM (Roussel Uclaf Causality Assessment Method) and updates, which provides a quantitative assessment of causality for cases of DILI and HILI, may be appropriate (Danan and Teschke, 2015).

For reports with multiple suspect drugs, including those with conventional medicines as co-suspected drugs, determining causality for the reported ADRs is more difficult. For this reason, the in-depth analysis focused on reports with a single herbal 


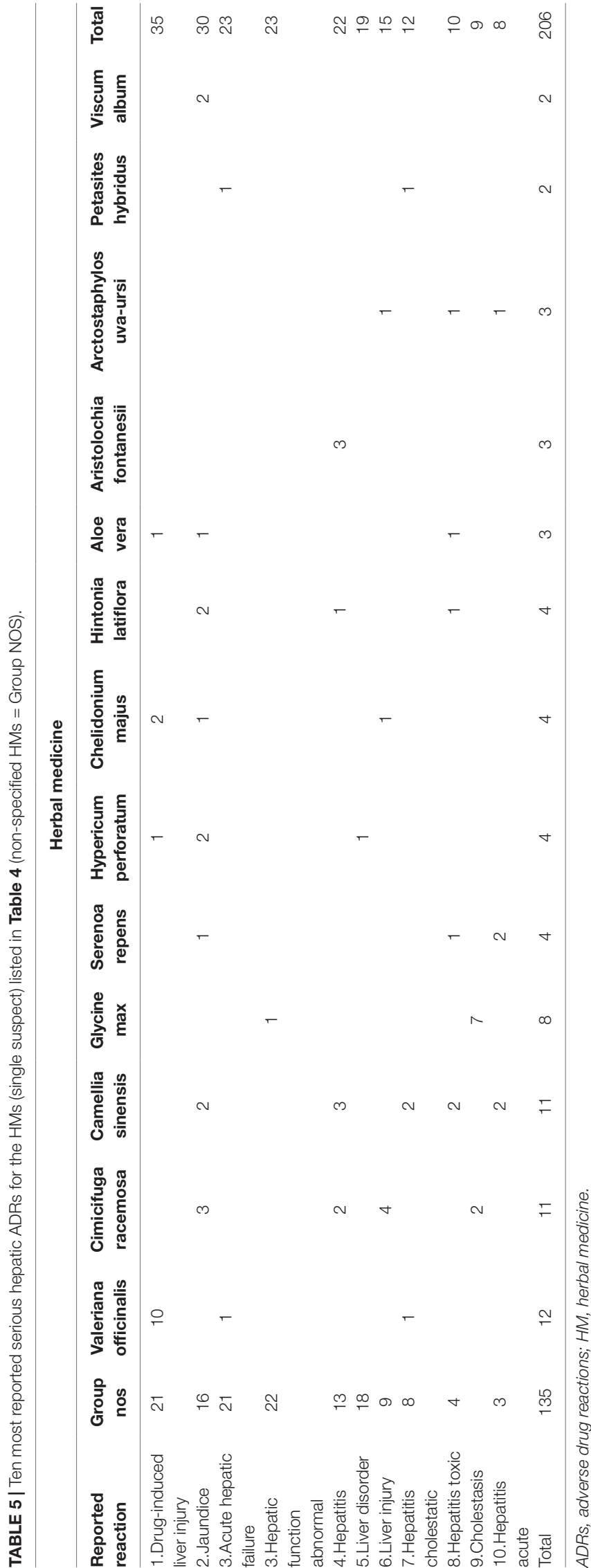

preparation as the suspect drug. However, such preparations can still comprise multi-herb mixtures, as described. A limitation of our approach is that many multi-herb preparations described in reports of $\mathrm{HBD}$ were excluded from the analysis, yet these products may pose the same, or an even greater, risk of causing HBD as do single-herb preparations. In the dataset, the HMs concerned comprised both finished (manufactured) herbal preparations (some with commercial names) and raw (crude) herbal materials. Further, data on the part of the herb used are not available in this dataset. This information is important as, for some plant species, different parts of the plant are used medicinally; the profile of chemical constituents in different in different plant parts, therefore they are considered to be different herbal drugs. Thus, we cannot exclude the possibility that the hepatotoxicity is related to a "wrong" part of the plant being used. Also, for finished products, we cannot exclude the possibility that counterfeit herbal ingredients were used.

This analysis found that, for a substantial portion of the HM reports in VigiBase, the preparations described are coded as "unspecified herbals"; this presents an important limitation in undertaking causality assessment for individual case reports or case-series and prevents use of these reports for signal detection purposes. Further, it can be challenging to store reports on "unspecified herbals" in a database without the fixed structure that is in place for regular drugs, with registered proprietary names, ingredients and the possibility to code on different levels. Using correct nomenclature is essential in order to precisely identify the herbal substance(s) implicated in ADR reports in a PV database, also for HMs where this can be complex such as TCM (Wu et al., 2007). The use of scientific binomial names, including botanical authority, is essential, in addition to naming the plant part used and preparation method in the reports, and subsequently, in VigiBase (Farah et al., 2000; Farah et al., 2006; Dauncey et al., 2019). However, for several reasons, it is often not possible to assign this information: ADR reports submitted by HMs' users and health professionals typically use common names for HMs (e.g., "echinacea"), which cannot be coded to a particular plant species and plant part; the herbal preparation used may be an unnamed traditional herbal formula comprising crude (e.g., dried, fragmented) herbal drugs; manufacturers' product labels also do not precisely define the plant species and parts used for herbal ingredients.

For PV centers that use the WHO Drug Global dictionary, the Herbal ATC (Anatomical Therapeutic Chemical), or HATC, coding system is available. The HATC classification aims to provide a scientific framework for a harmonized, global nomenclature and therapeutic classification of herbal substances and combinations of them (the Uppsala Monitoring Centre, 2017). However, the system has major limitations, and is not universally used. Limitations include that HMs may contain multiple ingredients and it is not always possible to identify them all. In addition, the HATC classification was developed for the whole plant, but not for a given part of the plant, which may be coded in several places in the HATC classification. Also, HMs from reporting countries may have only the vernacular name in the label of the product. Since VigiBase contains reports that have been transferred from local PV databases, it is essential that 
in the reporting, coding, assessing, storing, and transferring of HM reports essential information on the product is recorded.

Single suspect HMs for which serious hepatic reactions were most frequently reported were Cimicifuga racemosa (Actea racemosa, black cohosh), Valeriana officinalis, Camellia sinensis, and Hypericum perforatum. Black cohosh (Actea racemosa) has previously been associated with hepatotoxicity (Whiting et al., 2002; van de Meerendonk et al., 2009; Teschke, 2010; Teschke et al., 2011). However, cases described in the literature have confounding variables as well as poor quality data on the product implicated, ADR description, temporal association, co-morbidities, among others (Teschke, 2010) making causality assessment challenging (Teschke et al., 2009). Case descriptions of hepatotoxic reactions associated with Valeriana officinalis are more scarce (Cohen and Del, 2008; Vassiliadis et al., 2009; Douros et al., 2016). Also, for Hypericum perforatum, few cases of hepatotoxicity have been described (Dominguez Jimenez et al., 2007; Agollo et al., 2014). In a publication by Mazzanti et al, Camellia sinensis has been associated with nineteen cases of hepatotoxicity related to the consumption of herbal products containing green tea (Mazzanti et al., 2015). The same plant, Camellia sinensis, is used to produce all types of tea, and the differences among the various types arise from the different processing steps that are used. Based on the degree of fermentation, tea can be classified as black, green, white, or oolong tea. Of these, black tea is the most or fully fermented tea (Jolvis Pou, 2016).

In addition, the increasing international trade in HMs raises concerns around the safety and efficacy of these products (Walker and Applequist, 2012). There might be adulterated material in the supply chain of raw/crude herbal ingredients, or falsification in herbal products (Mishra et al., 2016). In this case, liver injury in relation to counterfeit herbal products may be due to the presence of plant species not declared on the product label. Strict legislation and quality control are needed. In addition, the development of crude drug repositories to maintain authentic botanicals as reference standards is essential (Srirama et al., 2017). The Royal Botanical Garden Kew for instance has a Chinese Herbal Medicine Authentication centre (Royal Botanical Gardens Kew, 2019).

\section{Strengths and Limitations}

A strength of this study is the advantage of presenting information from the global WHO ICSR database, where reports from all countries belonging to the international PV program are concerned. However, in VigiBase, the likelihood that the reported event was caused by the medicine varies from report to report. Some countries collect only suspected ADRs with at least a possibility of a causal relationship between the drug and reported event, while for example the United States, contributing almost half of the reports in VigiBase, collects "... any adverse event associated with the use of a drug in humans, whether or not considered drug related..." (GPO US Government Publishing Office, 2019). Because the data extracted from VigiBase itself had limitations in documentation, as mentioned above in our discussion, and the narrative of the individual ICSRs was not available, no causality assessment for individual cases was performed. The MedDRA codes for HBD do not provide (all) diagnostic information that might be present in the report. In addition, information on the dosage used in the reports was very incomplete and from our analysis of the data also seems incorrect on instance. Therefore, we decided not to use the data as it, unfortunately, would not be of value to the article. Considering the reported indications, jaundice was reported as the indication in $18 \%$ of the serious reports. However, it is difficult to ascertain if coding of the indications was done correctly at local PV centers.

As general limitations, we also point to the problem of underreporting ADRs in relation to HMs (Barnes, 2003; Walji et al., 2009; Skalli and Jordan, 2017), and the fact that methods and tools for causality assessment and signal detection have mostly been developed for conventional medicines and can be applied with difficulty to HMs. These need to be better adapted for a better and efficient phyto/herbovigilance.

\section{CONCLUSION}

This study explored reports of HBD associated with the use of HMs in the global ADR database VigiBase. Almost 2,500 reports for HMs and with at least one ADR coded to the MedDRA ${ }^{\circ}$ SOC HBD were retrieved from VigiBase; this number represents $0.013 \%$ of all reports in VigiBase. Many countries were underrepresented as contributors of reports, particularly those where use of herbal and other traditional medicines is a widely accepted or, in some cases, the only, form of healthcare available to millions of people. Substantial effort is required at the local, national, and international levels to raise awareness among users of HMs, herbal and traditional medicine practitioners, and healthcare professionals of the importance of considering, identifying and reporting cases of suspected ADRs associated with use of herbal and traditional medicines, and to facilitate access to reporting mechanisms, particularly in countries where a majority of the population relies on these preparations for their health and well-being.

Of the HBD SOC HM reports, around 25\% concerned a single herbal species as the suspect "drug." Substantial issues with coding of the suspect drugs were found. For single suspect HMs with multiple reports of HBD classed as "serious," in-depth causality assessment of the cases is needed to draw conclusions on the strength of the relationships.

\section{DATA AVAILABILITY STATEMENT}

The datasets for this manuscript are not publicly available because of the UMC data protection policy. The first author is responsible for the data being handled according to the UMC guidance for use of VigiBase data in studies. Requests to access the datasets should be directed to FH, f.vanhunsel@lareb.nl. 


\section{AUTHOR CONTRIBUTIONS}

FH, SK, SS, AK, J-BW, and JB contributed conception and design of the study. FH organized the dataset. SK, FH, and LT performed the (statistical) analysis; FH and SK wrote the first draft of the manuscript with input of all other authors. All authors contributed to manuscript revision, read, and approved the submitted version.

\section{REFERENCES}

Agollo, M. C., Miszputen, S. J., and Diament, J. (2014). Hypericum perforatuminduced hepatotoxicity with possible association with copaiba (Copaifera langsdorffii Desf):case report: Einstein.(Sao.Paulo) 12, 3,, 355-357. doi: 10.1590/s1679-45082014rc2953

Barnes, J. (2003). Pharmacovigilance of herbal medicines : a UK perspective: Drug Saf. 26 , 12, 829-851. doi: 10.2165/00002018-200326120-00001

Barnes, J., Abbot, N. C., Harkness, E. F., and Ernst, E. (1999). Articles on complementary medicine in the mainstream medical literature: an investigation of medline, 1966 through 1996: Arch. Intern. Med. 159, 15, 1721-1725. doi: 10.1001/archinte.159.15.1721

Barnes, J., Mills, S. Y., Abbot, N. C., Willoughby, M., and Ernst, E. (1998). Different standards for reporting ADRs to herbal remedies and conventional OTC medicines: face-to-face interviews with 515 users of herbal remedies: $\mathrm{Br}$. J. Clin. Pharmacol. 45, 5, 496-500. doi: 10.1046/j.1365-2125.1998.00715.x

Björnsson, E. S., Bergmann, O. M., Björnsson, H. K., Kvaran, R. B., and Olafsson, S. (2013). Incidence, presentation, and outcomes in patients with drug-induced liver injury in the general population of Iceland. Gastroenterology. 144, 14191425. doi: 10.1053/j.gastro.2013.02.006

Bunchorntavakul, C., and Reddy, K. R. (2013). Review article: herbal and dietary supplement hepatotoxicity. Aliment.Pharmacol.Ther 37p (1), 3-17. doi: 10.1111/apt.12109

Cohen, D. L., and Del, T. Y. (2008). A case of valerian-associated hepatotoxicity. J. Clin.Gastroenterol. 42 (8), 961-962. doi: 10.1097/MCG.0b013e3180500348

Danan, G., and Teschke, R. (2015). RUCAM in drug and herb induced liver injury: the update. Int. J. Mol. Sci. 17 (1), 1-33. doi: 10.3390/ijms17010014

Dauncey, E. A., Irving, J. T. W., and Allkin, R. (2019). A review of issues of nomenclature and taxonomy of Hypericum perforatum L. and Kew's Medicinal Plant Names Services. J. Pharm. Pharmacol. 71 (1), 4-14. doi: 10.1111/jphp. 12831

de Boer, A., Hunsel., F. van, and Bast, A. (2015). Adverse food-drug interactions. Regul.Toxicol Pharmacol. 73p (3), 859-865. doi: 10.1016/j.yrtph.2015.10.009

Dominguez Jimenez, J. L., Pleguezuelo, N. M., Guiote, M. S., Fraga, R. E., Montero Alvarez, J. L., and Poyato, G. A. (2007). [Hepatotoxicity associated with Hypericum (St. John's wort)]. Gastroenterol.Hepatol. 30 (1), 54-55. doi: 10.1157/13097456

Douros, A., Bronder, E., Andersohn, F., Klimpel, A., Kreutz, R., Garbe, E., et al. (2016). Herb-Induced Liver Injury in the Berlin Case-Control Surveillance Study. Br. J. Clin. Pharmacol. 79 (6), 988-99. doi: 10.1111/bcp.12565

Ekor, M. (2014). The growing use of herbal medicines: issues relating to adverse reactions and challenges in monitoring safety. Front.Pharmacol 4, 177. doi: 10.3389/fphar.2013.00177

Farah, H. M., Edwards, R., Linquist, M., Leon, C., and Shaw, D. (2000). International Monitoring of Adverse Health Effects Associated with Herbal Medicines. Pharmacoepidemiology Drug Saf. 9, 105-112. doi: 10.1002/ (SICI)1099-1557(200003/04)9:2<105::AID-PDS486>3.0.CO;2-2

Farah, M. H., Olsson, S., Bate, J., Lindquist, M., Edwards, R., Simmonds, M. S., et al. (2006). Botanical nomenclature in pharmacovigilance and a recommendation for standardisation. Drug Saf. 29 (11), 1023-1029. doi: 10.2165/00002018-200629110-00002

Fisher, P., and Ward, A. (1994). Complementary medicine in Europe. BMJ. 309 (6947,), 107-111. doi: 10.1136/bmj.309.6947.107

Garcia-Cortes, M., Robles-Diaz, M., Ortega-Alonso, A., Medina-Caliz, I., and Andrade, R. J. (2016). Hepatotoxicity by Dietary Supplements: A Tabular Listing and Clinical Characteristics. Int. J. Mol. Sci. 17 (4), 537. doi: 10.3390/ ijms 17040537

\section{ACKNOWLEDGMENTS}

The Global ICSR database VigiBase was used as a data source for this article. The information in VigiBase comes from a variety of sources, and the probability that the suspected adverse effect is drug-related is not the same in all cases. The information in this article does not represent the opinion of the UMC or the World Health Organization.

GPO US Government Publishing Office (2019). Electronic Code of Federal Regulations. - $\$ 314.80$ Postmarketing reporting of adverse drug experiences., https://www.ecfr.gov/cgi-bin/text-idx?SID=77821c5f7ae204cd5251ed82639d $876 \mathrm{a} \& \mathrm{mc}=$ true\&node=se21.5.314_180\&rgn=div8, < https://www.ecfr.gov/ $>$, Accessed May 23, 2019.

Guo, X. J., Ye, X. F., Wang, X. X., Wang, J., Shi, W. T., and Gao, Q. B., et al. (2015). Reporting patterns of adverse drug reactions over recent years in China: analysis from publications. Expert Opin. Drug Saf. 14 (2), 191-198. doi: 10.1517/14740338.2015.985647

Gupta, N., Vashist, P., Tandon, R., Gupta, S. K., Kalaivani, M., and Dwivedi, S. N. (2017). Use of traditional eye medicine and self-medication in rural India: A population-based study. PloS One 12 (8), e0183461. doi: 10.1371/journal. pone.0183461

Hazell, L., and Shakir, S. A. (2006). Under-reporting of adverse drug reactions: a systematic review. Drug Saf. 29 (5), 385-396. doi: 10.2165/00002018-200629050-00003

Jolvis Pou, K. R. (2016). Fermentation: The Key Step in the Processing of Black Tea. J. Biosyst. Eng. 41 (2), 85-92. doi: 10.5307/JBE.2016.41.2.085

Kemppainen, L. M., Kemppainen, T. T., Reippainen, J. A., Salmenniemi, S. T., and Vuolanto, P. H. (2018). Use of complementary and alternative medicine in Europe: Health-related and sociodemographic determinants. Scand.J Public Health 46 (4), 448-455. doi: 10.1177/1403494817733869

Linquist, M. (2008). VigiBase, the WHO Global ICSR Database System. Basic Facts.: Drug Inf. J. 42 (5), 409-419. doi: 10.1177/009286150804200501

Liu, S. H., Chuang, W. C., Lam, W., Jiang, Z., and Cheng, Y. C. (2015). Safety surveillance of traditional Chinese medicine: current and future. Drug Saf. 38 (2), 117-128. doi: 10.1007/s40264-014-0250-z

Mazzanti, G., Di, S. A., and Vitalone, A. (2015). Hepatotoxicity of green tea: an update. Arch. Toxicol. 89 (8), 1175-1191. doi: 10.1007/s00204-015-1521-x

Meyboom, R. H., Hekster, Y. A., Egberts, A. C., Gribnau, F. W., and Edwards, I. R. (1997). Causal or casual? The role of causality assessment in pharmacovigilance. Drug Saf. 17 (6), 374-389. doi: 10.2165/00002018-199717060-00004

Mishra, P., Kumar, A., Nagireddy, A., Mani, D. N., Shukla, A. K., Tiwari, R., et al. (2016). DNA barcoding: an efficient tool to overcome authentication challenges in the herbal market. Plant.Biotechnol.J 14 (1), 8-21. doi: 10.1111/pbi.12419

Navarro, V. J., Khan, I., Bjornsson, E., Seeff, L. B., Serrano, J., and Hoofnagle, J. H. (2017). Liver injury from herbal and dietary supplements. Hepatology 65 (1), 363-373. doi: doi.org/10.1002/hep.28813

Navarro, V. J., Barnhart, H., Bonkovsky, H. L., Davern, T., Fontana, R. J., Grant, L., et al. (2014). Liver injury from herbals and dietary supplements in the U.S. Drug-Induced Liver Injury Network. Hepatology 60 (4), 399-1408. doi: 10.1002/ hep. 27317

Necyk, C., Tsuyuki, R. T., Boon, H., Foster, B. C., Legatt, D., Cembrowski, G., et al. (2014) . Pharmacy study of natural health product adverse reactions (SONAR): a cross-sectional study using active surveillance in community pharmacies to detect adverse events associated with natural health products and assess causality. BMJ.Open 4 (3), e003431. doi: 10.1136/bmjopen-2013-003431

Ploen, M. (2018). Signal detection in a global database. https://www.meddra. org/sites/default/files/page/documents_insert/signal_detection_in_a_global_ database.pdf, <https://www.meddra.org/>, Accessed May 1, 2019.

Posadzki, P., Watson, L. K., and Ernst, E. (2013). Adverse effects of herbal medicines: an overview of systematic reviews. Clin.Med (Lond.) 13 (1), 7-12. doi: 10.7861/clinmedicine.13-1-7

Real, M., Barnhill, M. S., Higley, C., Rosenberg, J., and Lewis, J. H. (2019). DrugInduced Liver Injury: Highlights of the Recent Literature. Drug Saf. 42 (3), 365-387. doi: 10.1007/s40264-018-0743-2 
Royal Botanical Gardens Kew (2019). Chinese medicinal plants and their materia medica. https://www.kew.org/science/our-science/projects/chinese-medicinalplants-materia-medica, Accessed June 6, 2019.

Shaw, D., Graeme, L., Pierre, D., Elizabeth, W., and Kelvin, C. (2012). Pharmacovigilance of herbal medicine. J. Ethnopharmacol. 140 (3), 513-518. doi: 10.1016/j.jep.2012.01.051

Skalli, S., Zaid, A., and Soulaymani, R. (2007). Drug interactions with herbal medicines. Ther. Drug Monit. 29 (6), 679-686. doi: 10.1097/ FTD.0b013e31815c17f6

Skalli, S., and Soulaymani, B. R. (2012). Safety monitoring of herb-drug interactions: a component of pharmacovigilance. Drug Saf. 35 (10), 785-791. doi: 10.2165/11631790-000000000-00000

Skalli, S., and Bencheikh, R. S. (2015). Pharmacovigilance of herbal medicines in Africa: Questionnaire study. J. Ethnopharmacol. 171, 99-108. doi: 10.1016/j. jep.2015.05.033

Skalli, S., and Jordan, S. A., (2017). "Herbal and Traditional Medicines, Now and Future," in Pharmacovigilance Critique and Ways Forward. Eds. Edwards, R., and Lindquist, M. (Springer International Publishing) Cham, Switzerland, 145-159. doi: 10.1007/978-3-319-40400-4_14

Srirama, R., Santhosh Kumar, J. U., Seethapathy, G. S., Newmaster, S. G., Ragupathy, S., Ganeshaiah, K. N., et al. (2017). Species Adulteration in the Herbal Trade: Causes, Consequences and Mitigation. Drug Saf. 40p (8), 651661. doi: 10.1007/s40264-017-0527-0

Stournaras, E., and Tziomalos, K. (2015). Herbal medicine-related hepatotoxicity, 2015. World J. Hepatol. 7 (19), 2189-2193. doi: 10.4254/wjh.v7.i19.2189

Teschke, R. (2014). Traditional Chinese Medicine Induced Liver Injury. J. Clin. Transl. Hepatol. 2 (2), 80-94. doi: 10.14218/JCTH.2014.00003

Teschke, R. (2010). Black cohosh and suspected hepatotoxicity: inconsistencies, confounding variables, and prospective use of a diagnostic causality algorithm. A critical review. Menopause. 17 (2), 426-440. doi: 10.1097/ gme.0b013e3181c5159c

Teschke, R., Schwarzenboeck, A., Eickhoff, A., Frenzel, C., Wolff, A., and Schulze, J. (2013). Clinical and causality assessment in herbal hepatotoxicity. Expert Opin. Drug Saf. 12 (3), 339-366. doi: 10.1517/14740338.2013.774371

Teschke, R., Schwarzenboeck, A., Schmidt-Taenzer, W., Wolff, A., and Hennermann, K. H. (2011). Herb induced liver injury presumably caused by black cohosh: a survey of initially purported cases and herbal quality specifications. Ann. Hepatol 10 (3), 249-259. doi: 10.1016/S1665-2681(19)31536-4

Teschke, R., Bahre, R., Genthner, A., Fuchs, J., Schmidt-Taenzer, W., and Wolff, A. (2009). Suspected black cohosh hepatotoxicity-challenges and pitfalls of causality assessment. Maturitas 63 (4), 302-314. doi: 10.1016/j. maturitas.2009.05.006

The Council for International Organizations of Medical Sciences (CIOMS) (2001). CIOMS Current Challenges in Pharmacovigilance: Pragmatic Approaches. Report of CIOMS Working Group V. ., https://cioms.ch/wp-content/uploads/2017/01/ Group5_Pharmacovigilance.pdf, < https://cioms.ch >, Accessed April 26, 2019.

The International Conference on Harmonisation of Technical Requirements for Registration of Pharmaceuticals for Human Use (ICH) (2017). Welcome to MedDRA. https://www.meddra.org/, < https://www.meddra.org/>, Accessed June $16,2017$.

The R Foundation, and 2019, . The R Project for Statistical Computing. https:// www.r-project.org/, < https://www.r-project.org/>, Accessed April 26, 2019.

The SIDER Side Effect Resource. Hepatobiliary disease, 2015, http://sideeffects. embl.de/se/C0267792/, Accessed September 252019.

the Uppsala Monitoring Centre (2017). The Herbal Anatomical Therapeutic Chemical Classification System. https://www.who-umc.org/whodrug/ whodrug-portfolio/whodrug-global/herbal-atc/, Accessed May 31, 2017.

the Uppsala Monitoring Centre (2019). What is VigiBase. ?, https://www.who-umc. org/vigibase/vigibase/, < https://www.who-umc.org/>, Accessed May 1, 2019.

Tregunno, P. M., Fink, D. B., Fernandez-Fernandez, C., Lazaro-Bengoa, E., and Noren, G. N. (2014). Performance of probabilistic method to detect duplicate individual case safety reports. Drug Saf. 37 (4), 249-258. doi: 10.1007/ s40264-014-0146-y

Uppsala Monitoring Centre (2019). Caveat Document - Statement of reservations, limitations and conditions relating to data released from VigiBase, the WHO global database of individual case safety reports (ICSRs). https://www.who-umc. org/media/164610/umc_caveat.pdf, < https://www.who-umc.org/>, Accessed June 6, 2019.

US Food and Drug Administration (2019). FDA 101: Dietary Supplements. https:// www.fda.gov/consumers/consumer-updates/fda-101-dietary-supplements, Accessed September 25,

van de Meerendonk, H. W., van Hunsel, F. P., and van der Wiel, H. E. (2009). [Autoimmune hepatitis induced by Actaea racemosa. Side affects of an herb extract]. Ned.Tijdschr Geneeskd 153 (6), 246-249.

Vassiliadis, T., Anagnostis, P., Patsiaoura, K., Giouleme, O., Katsinelos, P., Mpoumponaris, A., et al. (2009). Valeriana hepatotoxicity. Sleep.Med 10 (8), 935. doi: 10.1016/j.sleep.2008.09.009

Venhuis, B. J., van Hunsel, F., van de Koppel, S., Keizers, P. H., Jeurissen, S. M., and De Kaste, D. (2016). Pharmacologically effective red yeast rice preparations marketed as dietary supplements illustrated by a case report. Drug Test Anal. 8 (3-4), 315-318. doi: 10.1002/dta.1929

Vickers, K. A., Jolly, K. B., and Greenfield, S. M. (2006). Herbal medicine: women's views, knowledge and interaction with doctors: a qualitative study. $B M C$ Complement.Altern.Med 6, 40. doi: 10.1186/1472-6882-6-40

Vohra, S., et al. (2012). Study of natural health product adverse reactions (SONAR): active surveillance of adverse events following concurrent natural health product and prescription drug use in community pharmacies. PloS One 7 (9), e45196. doi: 10.1371/journal.pone.0045196

Walji, R., Boon, H., Barnes, J., Austin, Z., Baker, G. R., and Welsh, S. (2009). Adverse event reporting for herbal medicines: a result of market forces. Healthc. Policy 4 (4), 77-90. doi: 10.12927/hcpol.2009.20820

Walker, K., and Applequist, W. L. (2012). Adulteration of Selected Unprocessed Botanicals in the U.S. Retail Herbal Trade: Economic Bot. 66 (4), 321-327. doi: 10.1007/s12231-012-9211-6

Wang, J. B., Zhu, Y., Bai, Z. F., Wang, F. S., Li, X. H., and Xiao, X. H. (2018a). Guidelines for the Diagnosis and Management of Herb-Induced Liver Injury. Chin.J Integr.Med 24 (9), 696-706. doi: 10.1007/s11655-018-3000-8

Wang, R., Qi, X., Yoshida, E.M., Méndéz-sánchez, N., Teschke, R., Sun, M., et al. (2018b). Clinical characteristics and outcomes of traditional Chinese medicine-induced liver injury: a systematic review. Expert Rev. Gastroenterol. Hepatol. 12 (4), 425-434. doi: 10.1080/17474124.2018.1427581

Whiting, P. W., Clouston, A., and Kerlin, P. (2002). Black cohosh and other herbal remedies associated with acute hepatitis. Med. J. Aust. 177 (8), 440-443. doi: 10.5694/j.1326-5377.2002.tb04886.x

Wong, M. C. S., Huang, J. L. W., George, J., Huang, J., Leung, C., Eslam, M., et al. (2019). The changing epidemiology of liver diseases in the Asia-Pacific region. Nat.Rev Gastroenterol.Hepatol. 16 (1), 57-73. doi: 10.1038/s41575-018-0055-0

World Health Organization, and 2013, WHO traditional medicine strategy: 2014-2023. https://www.who.int/medicines/publications/traditional/trm strategy14_23/en/, Accessed June 6, 2019.

World Health Organization, and 2019, Pharmacovigilance. https://www.who.int/ medicines/areas/quality_safety/safety_efficacy/pharmvigi/en/, <https://www. who.int/>, Accessed May 23, 2019.

World Health Organization (2004). WHO guidelines on safety monitoring of herbal medicines. 13-3-2007. Ref Type: Report.

Wu, K. M., Farrelly, J. G., Upton, R., and Chen, J. (2007). Complexities of the herbal nomenclature system in traditional Chinese medicine (TCM): lessons learned from the misuse of Aristolochia-related species and the importance of the pharmaceutical name during botanical drug product development. Phytomedicine 14 (4), 273-279. doi: 10.1016/j.phymed.2006.05.009

Conflict of Interest: The authors declare that the research was conducted in the absence of any commercial or financial relationships that could be construed as a potential conflict of interest.

Copyright $\odot 2019$ van Hunsel, van de Koppel, Skalli, Kuemmerle, Teng, Wang and Barnes. This is an open-access article distributed under the terms of the Creative Commons Attribution License (CC BY). The use, distribution or reproduction in other forums is permitted, provided the original author(s) and the copyright owner(s) are credited and that the original publication in this journal is cited, in accordance with accepted academic practice. No use, distribution or reproduction is permitted which does not comply with these terms. 